\title{
Quantitative assessment of salvaged myocardial zone and intramyocardial hemorrhage using non-contrast faster T2 mapping in a rat model by 7T MRI
}

\author{
YAN ZHANG ${ }^{1,2}$, YINI XU ${ }^{3}$, LEI WANG ${ }^{4}$, YUSHU CHEN ${ }^{4}$, RUIQING TIAN ${ }^{5}$, \\ JUN JIAO $^{1}$, HONG XIE $^{1}$, LI YANG $^{2}$ and FABAO GAO ${ }^{4}$
}

\begin{abstract}
${ }^{1}$ Department of Radiology, Affiliated Hospital of Guizhou Medical University, Guiyang, Guizhou 550004; ${ }^{2}$ Department of Radiology, General Hospital of PLA, Beijing 100853; ${ }^{3}$ The Key Laboratory of Optional Utilization of Natural Medicinal Resources, Guizhou Medical University, Huaxi University Town, Guiyang, Guizhou 550025; ${ }^{4}$ Molecular Imaging Laboratory, Department of Radiology, West China Hospital, Sichuan University, Chengdu, Sichuan 610041; ${ }^{5}$ Department of Oncology, The First People's Hospital of Guiyang, Guiyang, Guizhou 550002, P.R. China
\end{abstract}

Received July 5, 2016; Accepted March 24, 2017

DOI: $10.3892 /$ etm.2017.4967

\begin{abstract}
The aim of this study was to determine the myocardial area at risk (AAR), infarction-core size (IS) and the salvaged myocardial zone (SMZ), and to evaluate the imaging and histological characteristics of intramyocardial hemorrhage (IMH) after myocardial infarction using non-contrast T2 mapping on 7T magnetic resonance imaging (MRI). Twenty Sprague Dawley (SD) rats were randomly divided into the sham and model groups ( $\mathrm{n}=10$ in each). In the model group, myocardial infarction models were established by left anterior descending branch ligation. After $24 \mathrm{~h}$, all animals were imaged on a 7.0 Tesla system with cine spiral imaging, T2 mapping with late gadolinium enhancement (LGE). The rats were then sacrificed for measurement of the IS and AAR using 2,3,5-triphenylterazolium chloride (TTC) and hematoxylin and eosin (H\&E) staining. T2 mapping revealed that the AAR in the model group was significantly higher than that in the sham group. No remarkable T2 value was noted in the entire heart of the sham group. LGE and TTC staining demonstrated similar IS. T2 mapping and H\&E staining revealed a similar AAR as well. T2 mapping characterized the IMH as a phenomenon resulting from the area of hypointensity in the hyperintensity involving the infarct-core zone and corresponding $\mathrm{T} 2$ value
\end{abstract}

Correspondence to: Dr Li Yang, Department of Radiology, General Hospital of PLA, 28 Fu Xing Road, Beijing 100853, P.R. China

E-mail: yangli301@yahoo.com

Dr Fabao Gao, Molecular Imaging Laboratory, Department of Radiology, West China Hospital, Sichuan University, 37 Guo Xuexiang Road, Chengdu, Sichuan 610041, P.R. China

E-mail: gaofabao@yahoo.com

Key words: myocardial infarction, salvaged myocardial zone, intramyocardial hemorrhage, 7T MR imaging, rat model
$928.6 \pm 1.52 \mathrm{msec}$ with IMH vs. $35.8 \pm 2.61 \mathrm{msec}$ without IMH; $\mathrm{n}=3$ with 18 slices; $\mathrm{P}=0.032$ ). In conclusion, non-contrast T2 mapping was a reliable approach to quantitatively evaluate the SMZ and IMH.

\section{Introduction}

Myocardial area at risk (AAR) is defined as the extent of ischemic myocardium following coronary artery occlusion, which comprises the nonviable infarction-core size (IS) and the viable salvaged myocardial zone (SMZ). Thus, measurement of AAR and IS allows for determination of the SMZ, which is accepted as a stronger predictor of therapeutic efficacy after myocardial infarction (1). Moreover, intramyocardial hemorrhage (IMH) constantly emerges during the development of myocardial infarction, which subsequently induces corresponding alterations in T2 value on T2 maps, inducing subsequent injuries and elevating the morbidity and mortality (2-4). However, the imaging characteristics on T2 maps need to be illustrated.

During the past decades, multiple indirect strategies, such as nuclear imaging and echocardiography, have been introduced to evaluate myocardial viability. Unlike those approaches with the limitations of acoustic windows and radiation, cardiovascular magnetic resonance imaging (CMR) does not employ ionizing radiation and offers higher spatial and temporal resolution. Furthermore, CMR may quantitatively assess cardiac function, and its application for assessing viability is widely accepted (5-8). Conventional application of T2-weighted magnetic resonance imaging (MRI) to delineate the AAR, a dark blood turbo spin echo (TSE) technique has been employed to quantitatively measure the AAR and SMZ post-reperfusion (9), and T2-weighted MRI has been utilized to identify the incidence of myocardial edema. However, these techniques have certain limitations with regard to adaptation of image variations associated with respiratory or cardiac motion, the signal associated with surface coil intensity inhomogeneity and the effect of blood flow (10). In 2007, T2 mapping was introduced to assess the AAR at the early stage (11-13) and 
was subsequently performed in patients with myocardial infarction (14). Late gadolinium enhancement imaging (LGE) may be utilized to identify myocardial infarction characterized as increased signal intensity due to cellular membrane rupture and gadolinium-diethylenetriamine pentaacetic acid (Gd-DTPA) diffusion into intracellular spaces. Furthermore, a previous study by our group suggested that if myocardial edema were identified by novel faster T2 mapping, the AAR could be determined in spite of rats having an increased heart rate (15). Combined use of T2 mapping and LGE delineates the SMZ in ischemic myocardium and defines IMH as the area of hypointensity in the hyperintensity of the infarct-core zone.

The present study was designed to establish a stable and reliable platform using a non-invasive technique to assess the SMZ and IMH in a rat model despite a high heart rate.

\section{Materials and methods}

Study design. A total of 20 4-week-old male Sprague Dawley (SD) rats with a mean weight of $(250 \pm 20 \mathrm{~g})$ provided by the Experimental Animal Center of Guiyang Medical University (Guiyang, China) were included in the present study and randomly assigned to the model and sham groups $(\mathrm{n}=10)$. The rats were accustomed at $20^{\circ} \mathrm{C}, 70 \%$ humidity and 12 -h light/dark cycle with free access to food and water. In the model group, the animal models with myocardial infarction were established by left anterior descending branch (LAD) ligation as previously described (16). The animals were anesthetized by intraperitoneal injection of sodium pentobarbital (Sichuan Kelun Pharmaceutical Co., Ltd., Sichuan, China) at a dosage of $50 \mathrm{mg} / \mathrm{kg}$. Subsequently, the rats underwent thoracotomy and mechanical ventilation was monitored by real-time electrocardiogram (ECG). The LAD ligation was closed using a 7-0 silk suture. In the sham group, the animals received thoracotomy without LAD occlusion. The present study was approved by the Institutional Animal Research Committee of the First People's Hospital of Guiyang and was performed according to the recommendations of the Guide for the Care and Use of Laboratory Animals of the First People's Hospital of Guiyang (Guiyang, China).

MRI protocols. General. All MRI was performed on a 7T MRI (BioSpec 70/30; Bruker, Karlsruhe, Germany) 24 h after the corresponding treatments. First, cardiac function was assessed by cine spiral imaging of the entire left ventricle (LV), followed by $\mathrm{T} 2$ mapping imaging and LGE imaging. In each group, three rats were sacrificed and the heart was collected for 2,3,5-triphenylterazolium chloride (TTC) staining. Subsequently, the heart tissue was prepared for histological examination. Upon CMR, the rats were anesthetized with isoflurane (5 vol\%; Sichuan Kelun Pharmaceutical Co., Ltd.) in a 2:1 mixture of air $(0.3 \mathrm{l} / \mathrm{min})$ and oxygen $(0.1 \mathrm{l} / \mathrm{min})$, and then maintained under anesthesia (1.5-2.5 vol\% isoflurane) in a custom-built cradle to maintain a stable cardiac and respiratory rate. Each rat was placed in the prone position on a surface coil. ECG signal, respiratory and heart rates were obtained from three subcutaneous copper needles loaded in the bilateral hind limbs.

Novel faster T2 mapping. After maintaining the respiratory rate between 30 and 50 cycles per minute, novel faster
T2 mapping imaging was performed by changing the concentration of isoflurane. Five single-sliced simplified T2-mapping images were obtained on the short-axis slices during the mid-diastolic and end-inspiratory phases using ECG and respiratory gating systems. Imaging parameters were as follows: Repetition ime/echotime (TR/TE), 1,500 msec/10, 20, $30 \mathrm{msec}$; data acquisition time, $44 \mathrm{msec}$; matrix size, 192x192; field of view (FOV), $50 \times 50 \mathrm{~mm}$; and slice thickness, $1.5 \mathrm{~mm}$. The last TE of $30 \mathrm{msec}$ was selected based on the approximate $\mathrm{T} 2$ value of myocardial tissue at 7T MR. Analysis of variance (ANOVA) test was used for determination of AAR by novel faster T2 mapping.

LGE imaging. A standard multi-slice short axis late gadolinium fast low-angle shot-cine sequence was performed and cine MR images were acquired from the base to the apex. The imaging sequence was as follows: TR/TE, $8 \mathrm{msec} / 3.1 \mathrm{msec}$; flip angle, 10; matrix size, 256x256; FOV, 50x50 mm; slice thickness, $1.5 \mathrm{~mm}$; and 25 frames for each slice. Contrast enhancement was examined $10 \mathrm{~min}$ after intravenous administration of Gd-DTPA via the hind limbs $(0.2 \mathrm{mmol} / \mathrm{kg}$; Magnevist, Bayer Health Care Pharma AG, Berlin, Germany) and anesthetics (1.5-2.5 vol\% isoflurane) in a custom-built cradle were used. The imaging parameters were TR/TE, $5.2 \mathrm{msec} / 1.8 \mathrm{msec}$; flip angle, 25; matrix size, 256x256; FOV, $50 \times 50 \mathrm{~mm}$; slice thickness, $1.5 \mathrm{~mm}$, and 25 frames for each slice. All CMR parameters are illustrated in Table I.

CMR imaging. Cardiac function at the end-diastolic and end-systolic phases was evaluated using cine spiral imaging, and the LV borders were manually delineated and calculated by multiplying the size involving slice thickness and gap. T2 mapping imaging data were analyzed using specific software in Matlab 7.1 (Mathwork Inc., Natick, MA, USA). The $\mathrm{T} 2$ value was obtained by manually drawing the region of interest (ROI), $\sim 0.06 \mathrm{~cm}^{2}$ (88 pixels) on T2 maps from the base to apex with a total of 6 slices. The AAR was then defined as the area of ischemic myocardium with a $\mathrm{T} 2$ value standard deviation (SD) of $>2$ from the remote myocardium on T2 mapping, and the infarction area was defined with a signal intensity SD of $>5$ above the mean of remote myocardium on LGE images, both of which were calculated by manual delineation of the square centimeter. Thereafter, the SMZ was obtained by subtracting the infarction size by LGE from the AAR. The IMH was identified as the area of hypointensity within the hyperintensity infarction area on T2 maps. The AAR, IS and SMZ were all expressed as the percentage of LV after parameter standardization. All manual data were independently measured by two experienced radiologists who were blinded to the study.

Histopathological analysis. After CMR imaging, the heart samples were prepared as paraffin-embedded slices (5- $\mu \mathrm{m}$ thick sections), and each slice was incubated with $1 \%$ 2,3,5-triphenyltetrazolium chloride (Sigma-Aldrich; Merck $\mathrm{KGaA}$, Darmstadt, Germany) for $15 \mathrm{~min}$ at $37^{\circ} \mathrm{C}$ and then observed under a microscope (magnification, x200). The infarction area was stained white, whereas the viable myocardium was stained red. LGE and TTC outcomes were visually matched by comparing in vivo images and ex vivo slices by two 
Table I. Acquisition parameters of the CMR methods.

\begin{tabular}{|c|c|c|c|c|c|c|c|c|c|}
\hline Method & $\begin{array}{c}\mathrm{TR} \\
(\mathrm{msec})\end{array}$ & $\begin{array}{c}\mathrm{TE} \\
(\mathrm{msec})\end{array}$ & FA & $\begin{array}{l}\text { Slice thickness } \\
(\mathrm{mm})\end{array}$ & $\begin{array}{l}\text { Reconstructed } \\
\text { matrix }\end{array}$ & FOV & NEX & $\begin{array}{c}\mathrm{TA} \\
(\mathrm{sec})\end{array}$ & $\begin{array}{l}\text { Acquisition } \\
\text { time (sec) }\end{array}$ \\
\hline LG-FLASH-cine & 8 & 3.1 & 10 & 1.5 & $256 \times 256$ & $5 \times 5$ & 1 & 51 & 51 \\
\hline Faster T2 mapping & 1500 & $10 / 20 / 30$ & - & 1.5 & $192 \times 192$ & $5 \times 5$ & 1 & 216 & 300 \\
\hline FISP-cine & 5.2 & 2 & 2.5 & 1.5 & - & $5 \times 5$ & 1 & 67 & 180 \\
\hline
\end{tabular}

FISP, fast imaging with steady state precession; LG-FLASH, late gadolinium fast low angle shot; TE, echo time; TR, repetition time; FA, fractional anisotropy; NEX, number of excitations; FOV, field of view; TA, time to appearance.

experienced radiologists blinded to the study. Stratified analysis was performed according to whether IMH was present, which was assessed by measuring the area of hypointensity within the hyperintensity infarction area in T2 maps. Histological sections were subjected to hematoxylin and eosin (H\&E) staining.

Statistical analysis. Values are expressed as the mean \pm standard error of the mean. Comparison between pairs of groups was performed using an independent-samples t-test. The correlation between LGE and TTC staining, T2 mapping and H\&E staining, and LGE and H\&E staining was analyzed by linear regression analysis using the Pearson coefficient $r$. ANOVA was used for determination of AAR by novel faster T2 mapping. The detection of AAR and IS between two groups was statistically compared using the paired t-test. $\mathrm{P}<0.05$ was considered to indicate a statistically significant difference.

\section{Results}

Animal grouping. The variations in the respiratory gating and ECG throughout the process of CMR affected the increase in time to acquire images. Sixteen rats were included in the final analysis. Two rats were excluded from the sham group due to low-quality images caused by motion artifacts. In the model group, one rat that died during anesthesia was excluded, and the other rat was excluded due to low-quality images due to technical reasons. In addition, three rats in the model group yielded hemorrhage according to the T2 maps.

CMR and histological assessment of myocardial infarction models. As illustrated in Table I and Fig. 1, the average end-diastolic volume (EDV) in the sham group was 125.6 $\pm 5.27 \mu 1$, which was significantly higher compared with $82.4 \pm 5.03 \mu 1$ in the model group ( $\mathrm{P}=0.004 ; \mathrm{n}=16)$ (Fig. 2). As illustrated in Fig. 3, the LGE images indicated a clear IS as a hypointensity region involving cardiac muscle by drawing a region (yellow dotted line; Fig. 3B), which was perfectly matched by TTC staining (blue dotted line; Fig. 3C). The IS was stained red, distinguishable from viable myocardium stained white. T2-mapping images (Fig. 3D) with three ETs of 10, 20 and $30 \mathrm{sec}$ revealed that the T2 value was in pseudo color (Fig. 3E). All T2 maps were scaled between 0 and $80 \mathrm{msec}$. The AAR is shown in the simplified T2 maps (red dotted line; Fig. 3F), which corresponded to the T2 maps (red dotted line; Fig. 3G). In an overlay of Fig. 3E and $\mathrm{F}$, the SMZ was calculated by subtracting the IS from

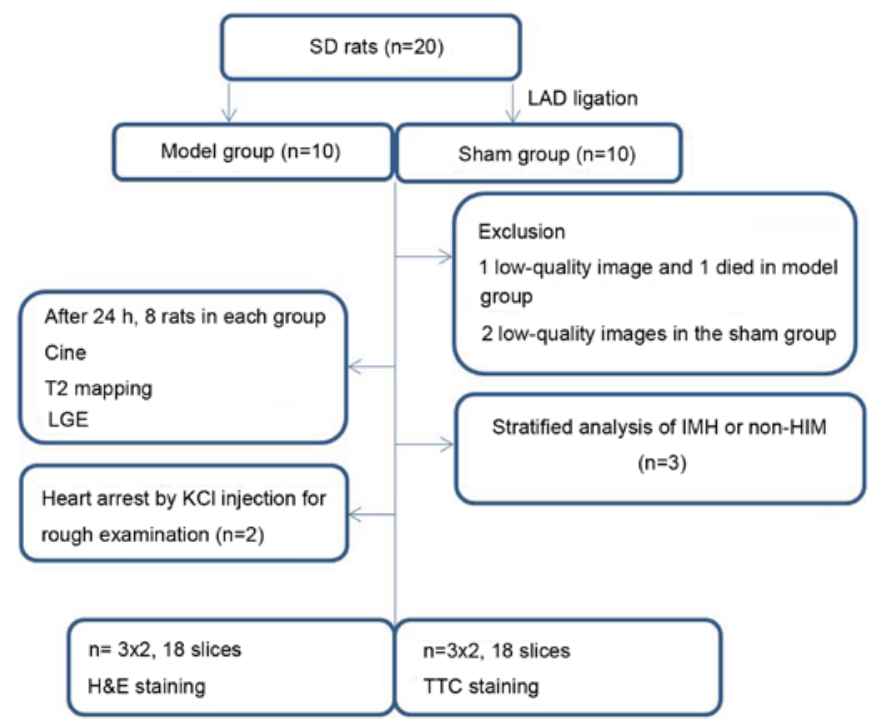

Figure 1. Flow chart of the study protocol. LAD, left anterior descending branch; Gd-DTPA, gadolinium-diethylenetriamine pentaacetic acid; LGE, late gadolinium enhancement imaging; TTC, 2,3,5-triphenylterazolium chloride staining; H\&E, hematoxylin and eosin staining; IMH, intramyocardial hemorrhage; SD, Sprague Dawley.

the AAR and is presented between the red and yellow dotted lines (Fig. 3G).

Determination of AAR by novel faster T2 mapping. ANOVA revealed that the AAR in the model group defined by novel faster T2 mapping was $38.6 \pm 1.52 \mathrm{msec}$, which was significantly higher than $26.8 \pm 1.48 \mathrm{msec}$ in the sham group $(\mathrm{P}=0.007)$; however, no significant difference was observed between the base and apex $\mathrm{T} 2$ values in the sham group $(\mathrm{P}=0.056)$. In the model group, the mean AAR, IS and SMZ were $38.6 \pm 1.52 \mathrm{msec}(\mathrm{P}=0.039), 42.4 \pm 1.67 \mathrm{msec}(\mathrm{P}=0.002)$ and $35.8 \pm 1.31 \mathrm{msec}(\mathrm{P}=0.016)$ compared with that in the sham group, as illustrated in Fig. 4A.

As shown in Fig. 4B, the proportion of AAR determined by $\mathrm{T} 2$ mapping had a good correlation with that determined by $H \& E$ staining. A statistical significance was noted between AAR determined by T2 mapping $(61.98 \pm 10.34 \%)$ and $\mathrm{H} \& \mathrm{E}$ staining (60.28 $\pm 9.53 \% ; \mathrm{n}=3$ with 18 slices; $\left.\mathrm{P}=0.0062 ; \mathrm{R}^{2}=0.96\right)$.

Agreement of IS determined by LGE and TTC. As shown in Fig. 5, the proportion of IS by LGE and TTC staining was in good correlation, indicating a significant difference 

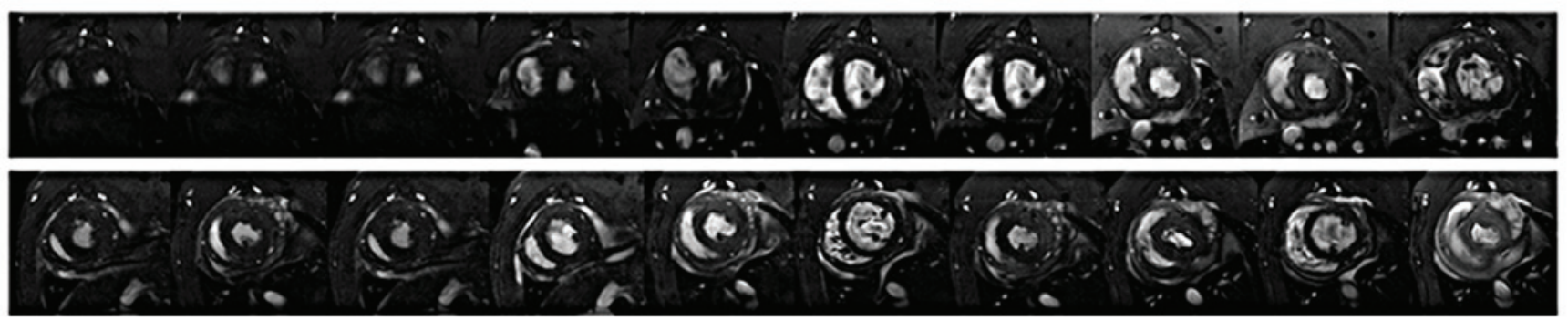

$\operatorname{Apex}(A)$

Base (B)

\section{FISP-Cine}

Figure 2. Assessment of the rat models with myocardial infarction. Comparison of magnetic resonance images from apex to base at $48 \mathrm{~h}$ between the (A) sham and (B) model groups. All images were selected from the base to apex by FISP-cine of all 25 frames in one cine cycle. The borders of left ventricles were manually delineated and calculated by multiplying the size. FISP, fast imaging with steady state precession.

A

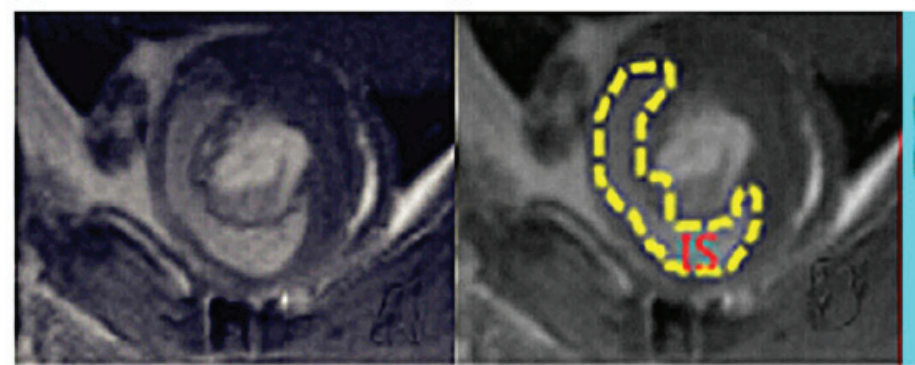

LGE

D
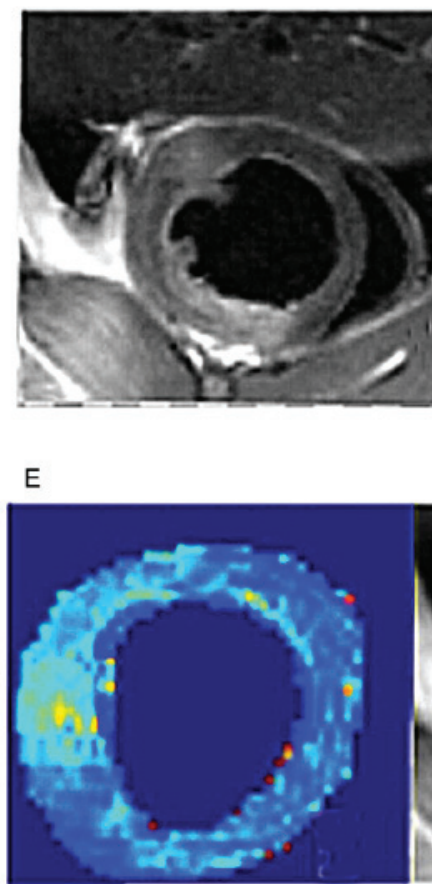

Novel faster T2-mapping

F

G

C

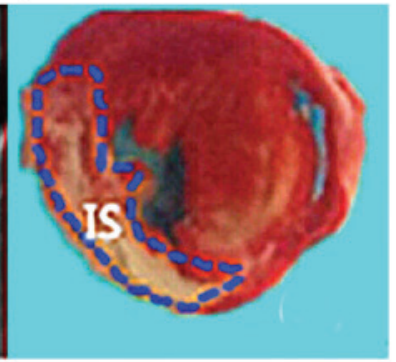

TTC
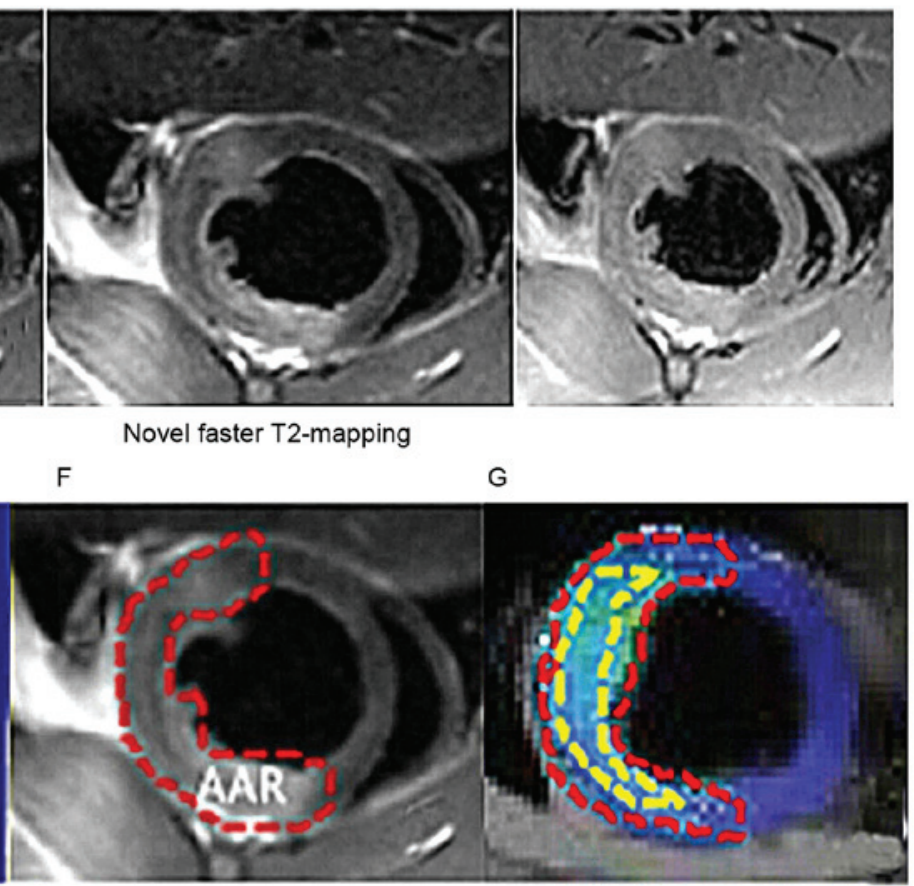

T2 maps

Figure 3. Magnetic resonance images obtained by combination of faster T2-mapping and LGE vs. histological examination. IS was clearly denoted as an extensive hyperintense region on (A) LGE, obtained by (B) drawing a region (yellow dotted line), which perfectly matched with (C) the TTC image (blue dotted line). (D) T2-mapping images with three echo times of 10, 20 and $30 \mathrm{sec}$, yielding (E) T2 value in pseudo color; all T2 maps were scaled between 0 and 80 msec. (F) The AAR was shown on the simplified T2 maps (red dotted line), corresponding to (G) the T2 map (red dotted line) in an overlay of (E) and (F). SMZ was presented between the zones within the red and yellow dotted lines. LGE, late gadolinium enhancement imaging; TTC, 2,3,5-triphenylterazolium chloride staining; AAR, area at risk; IS, infarction size.

in terms of IS between LGE and TTC staining $(47.99 \pm 10.56$ vs. $46.26 \pm 9.94 ; \mathrm{n}=3$ with 18 slices; $\left.\mathrm{P}=0.0064 ; \mathrm{R}^{2}=0.96\right)$.
Determination of SMZ by novel faster T2 mapping. The SMZ size was expressed as the percentage of LV for parameter 
A

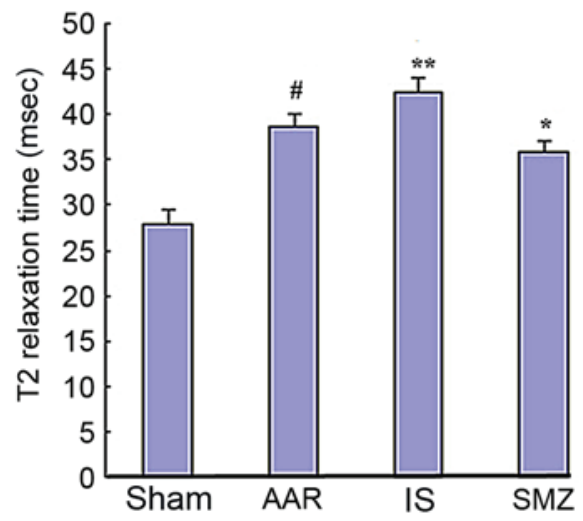

B

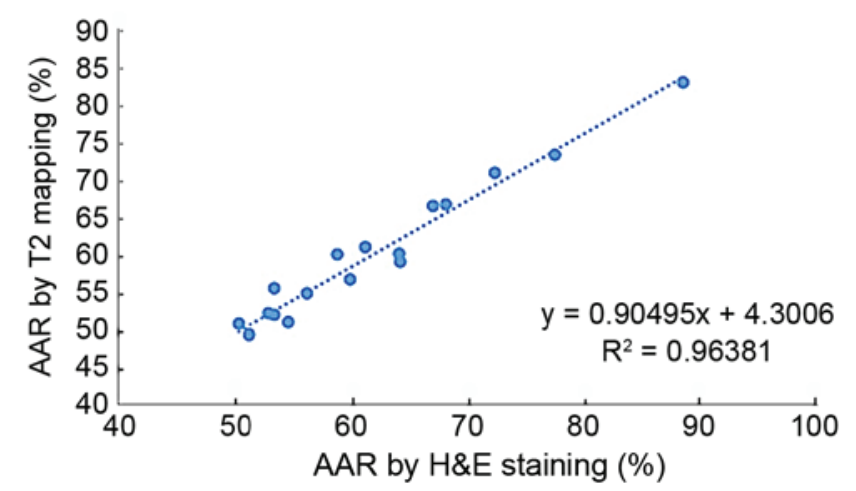

Figure 4. (A) Representative T2 relaxation time in the ischemic region. Analysis of variance was used for determination of AAR by novel faster T2 mapping. ${ }^{* *} \mathrm{P}<0.01,{ }^{*} \mathrm{P}<0.05$ vs. AAR; ${ }^{\mathrm{P}} \mathrm{P}<0.05$ vs. sham. (B) Slice-by-slice comparison of myocardial AAR by T2 mapping and histological examination. AAR, area at risk; IS, infarction size; SMZ, salvaged myocardium control: Remote infarction; H\&E, hematoxylin and eosin staining; MIC, myocardial infarction.

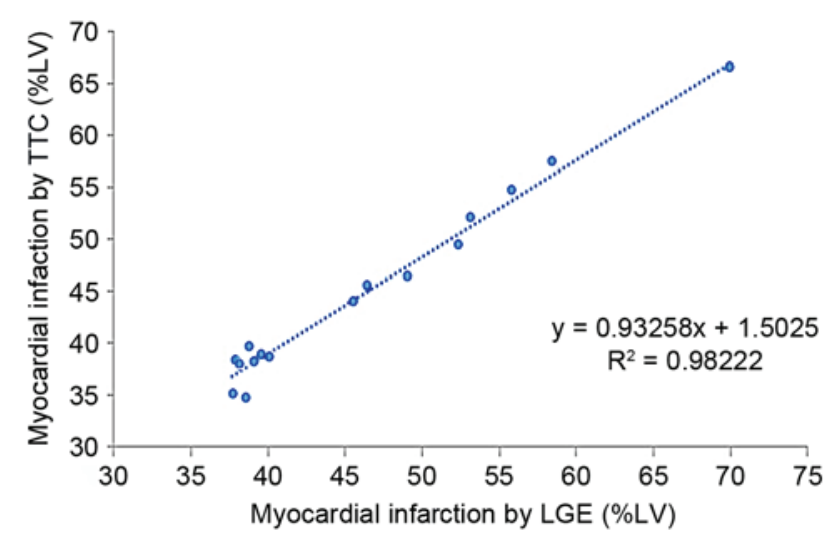

Figure 5. Slice-by-slice comparison of IS by LGE and TTC staining. LGE, late gadolinium enhancement imaging; TTC, 2,3,5-triphenylterazolium chloride staining; AAR, area at risk; IS, infar ction size; $\mathrm{LV}$, left ventricle.

standardization and determined using three methods. The SMZ was $13.48 \pm 3.64 \%$ of the LV (range, $7.72-20.6 \%$ ) according to quantification by T2 mapping and $13.04 \pm 3.68 \%$ of the LV (range, 7.81-20.5\%) as determined by AAR minus IS, which was consistent with $13.68 \pm 9.94 \%$ of the LV (range, 7.85-21.5\%; $\mathrm{n}=3$ with 18 slices; $\mathrm{P}=0.0068$ and $\mathrm{P}=0.0067, \mathrm{R}^{2}=0.95$ and 0.94 ) as assessed by H\&E staining (Fig. 6).

In vivo and ex vivo quantification of IMH. Intramyocardial hemorrhage was observed in three rats from the model group at $24 \mathrm{~h}$. IMH occurred after infarction corresponding to the T2 maps, which were surrounded by IS (green area; Fig. 7A). These characteristics referred to the 'patchy' hypointensity in the hyperintensity zone (white dotted line), as illustrated in Fig. 7A. Rough examination of the heart also confirmed the presence of IMH (red dotted line) in Fig. 7B, which was consistent with the findings of H\&E staining (blue dotted line) in Fig. 7C, histopathological findings in Fig. 7D (blue dotted line) and E. On T2-mapping images, IMH was characterized as the area of hypointensity in the hyperintensity infarction zone (Fig. 7A), accompanied by the following changes in the T2 value: $35.8 \pm 1.52 \mathrm{msec}$ with $\mathrm{IMH}$ and $32.2 \pm 1.92 \mathrm{msec}$ without IMH $(\mathrm{n}=3 ; \mathrm{P}=0.035)$.

\section{Discussion}

Myocardial AAR is defined as the incidence of ischemic myocardium after myocardial infarction, which consists of non-viable IS and viable SMZ. Consequently, measurement of AAR and IS allows for the determination of SMZ, which is recognized as a convincing predictor of viable myocardium and therapeutic efficacy after myocardial infarction (8-18).

The evidence from the present study indicated that combined application of in vivo non-contrast T2 mapping by MRI and LGE allows for accurate quantification of multiple ischemic components, including the AAR, IS and SMZ in a rat model. The SMZ detected by novel faster T2 mapping was independently correlated with histological outcomes. In addition, a 'patchy' area was found due to the hypointensity attenuation in the hyperintensity infarction-core zone on T2 maps, which was subsequently confirmed by H\&E staining.

Ischemic heart disease has been proven to be associated with poor function of LV, and cine MRI provides essential data and pivotal insights into LV function (19). Conventional techniques, such as Doppler or ultrasound and echocardiography in canine models or humans, have been used to assess myocardial function. In the present study, cine MRI provided EDV data of the LV to facilitate the real-time evaluation of the regional function, although the rats exhibited a relatively high heart rate.

The T2 value in the model group was significantly higher compared with that in the sham group. The present as well as a previous study by our group demonstrated no significant difference in the sham group from the base to apex on the T2 maps (15). However, these results were not consistent with the findings by other studies (20), probably due to differences in experimental animals and recruitment of human subjects. In the rat models with myocardial infarction, evaluation of T2 mapping resulted in AAR, IS and SMZ of 38.6 $\pm 1.52,42.4 \pm 1.67$ and $35.8 \pm 1.31 \mathrm{msec}$, corresponding to different $\mathrm{T} 2$ values.

The AAR refers to the signs of edema induced by myocardial injury, which includes SMZ and IS. The SMZ is a dynamic parameter and depends upon the delineation of AAR and IS. SMA also serves as a vital predictor of ensuing myocardial infarction (21-23). Conventionally, T2-weighted MRI has been 

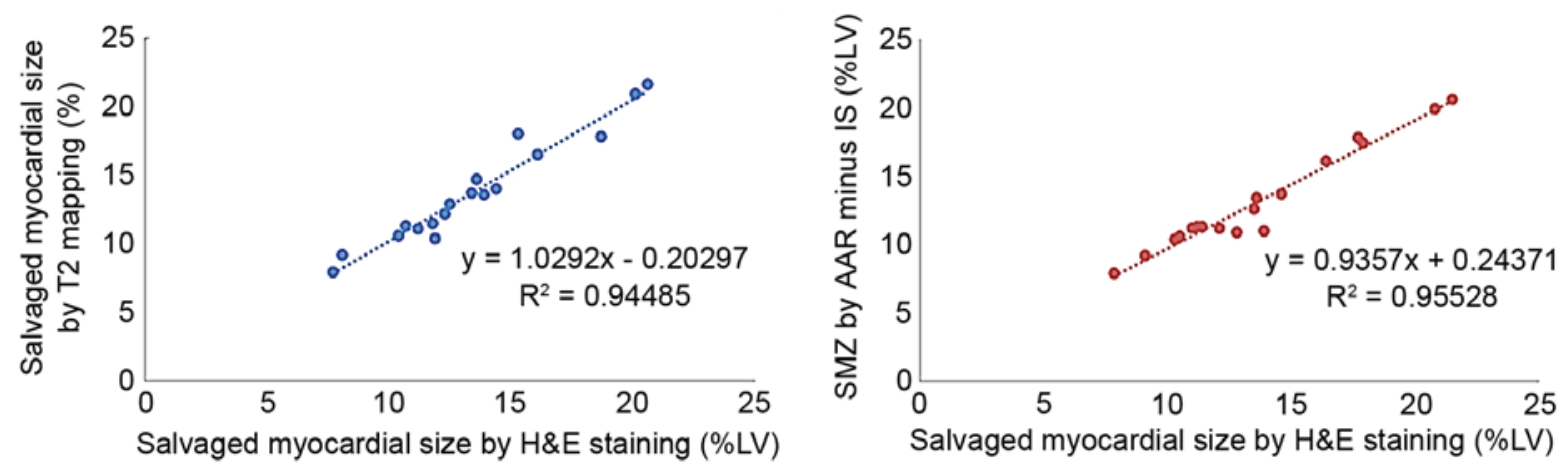

Figure 6. SMZ assessed by T2 mapping imaging compared with H\&E staining. SMZ, salvaged myocardium control: Remote infarction; H\&E, hematoxylin and eosin staining; AAR, area at risk; IS, infarction size; LV, left ventricle.
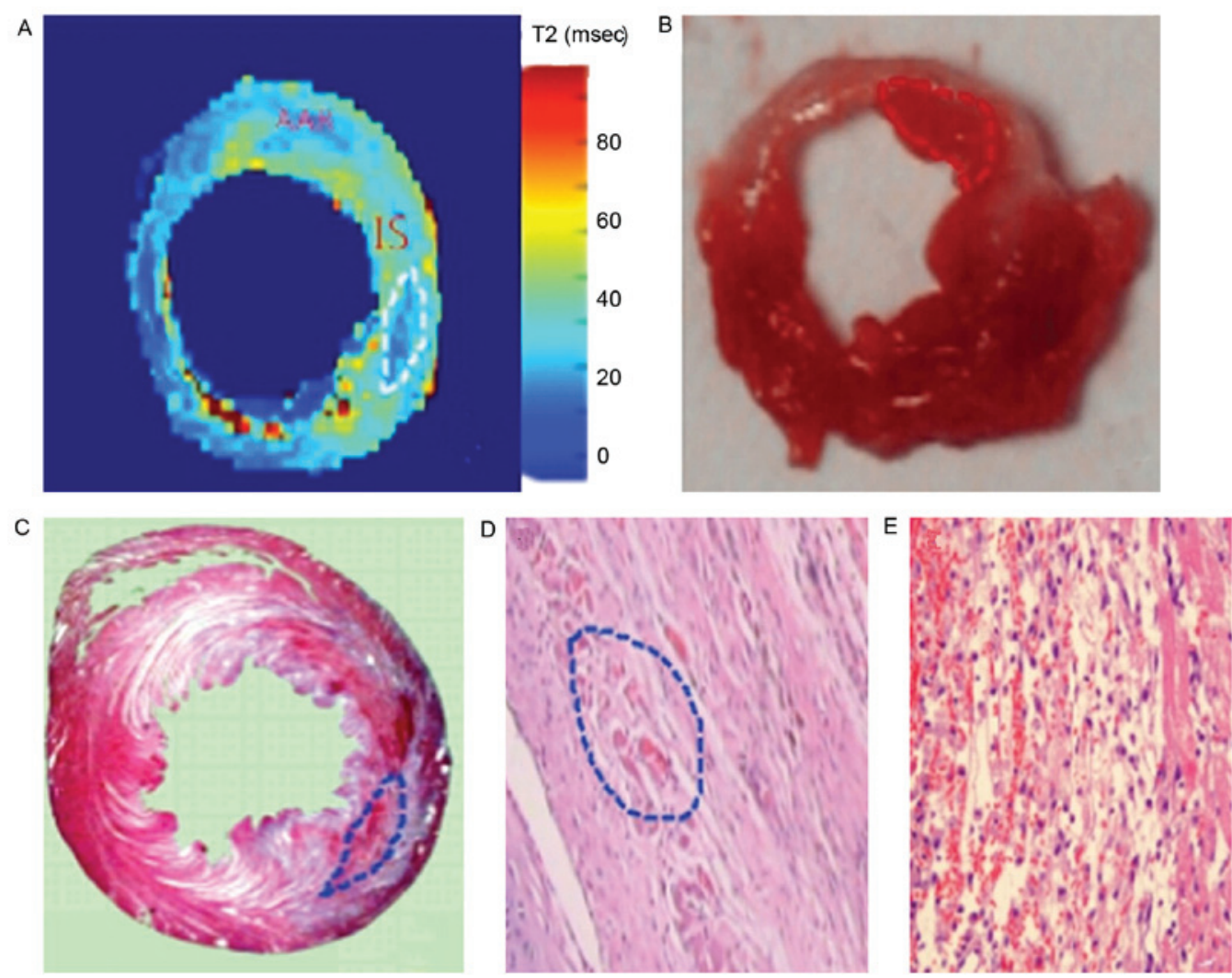

Figure 7. Assessment of IMH by T2 mapping vs. histological examination. (A) T2 mapping demonstrated that the IMH (white dotted line) was surrounded by IS (green area), corresponding to the 'patchy' hypointensity in the hyperintensity zone. (B) Rough examination of the heart tissue confirmed the incidence of IMH (red dotted line), perfectly matching with (C) the H\&E staining (blue dotted line), and (D and E) the histological analysis [blue dotted line; magnification, $\mathrm{x} 100$ in (D) and x200 in (E)]. H\&E, hematoxylin and eosin; IMH, intramyocardial hemorrhage; IS, infarction size.

employed to delineate and assess the AAR after myocardial infarction as a dark blood TSE technique. However, all of these approaches possess disadvantages, such as difficulty in adapting images to respiratory or cardiac motion and the signal associated with surface coil intensity inhomogeneity (24). T2 mapping has been first utilized to assess myocardial edema without the above limitations in $2007(25,26)$ and subsequently applied in patients diagnosed with myocardial infarction (27). In the present study, AAR was evaluated by a novel faster T2 mapping with three ETs of 10, 20 and $30 \mathrm{sec}$, and a T2 value in pseudo color was documented. These results represented an apparent linear correlation between AAR determined by faster T2 mapping and H\&E staining.
IS is another pivotal parameter for the evaluation of SMZ following myocardial infarction. LGE has been confirmed to possess a relatively high sensitivity of up to $99 \%$ for the evaluation of myocardial infarction, myocardial fibrosis and scars (28). In the present study, IS assessed by LGE was correlated with TTC staining. However, LGE was slightly overestimated in the actual infarction zone, which may explain for the peri-infarction zone being surrounded by edema after acute myocardial infarction, resulting in increased signal intensity. Of note, LGE has several limitations due to gadolinium dispersion, which is dependent on the time of image acquisition and dose of contrast agent $(29,30)$, which was observed in the process throughout the present 
study. Thus, in the present investigation, the experimental procedures were performed at $10 \mathrm{~min}$ after injection of $0.2 \mathrm{mmol} / \mathrm{kg}$ Gd-DTPA.

In the present study, three approaches were utilized to determine different parameters of the myocardial size at risk. Previous studies have stated that the SMZ is a time-dependent parameter, considered a vital predictor of poor prognosis induced by ventricular arrhythmia $(31,32)$. According to a mathematical equation, SMZ was calculated by subtracting IS from AAR. The SMZ calculated from the AAR minus the IS determined by LGE was slightly smaller than that assessed by histological examination, probably resulting from the abovementioned limitation of LGE. However, the SMZ assessed by faster T2 mapping perfectly matched with the findings of H\&E staining, suggesting that T2 mapping is a feasible technique to determine SMZ.

$\mathrm{IMH}$ is considered a sign of severe injury induced by vascular cell damage (33). By establishing in vivo rat models with cardiac infarction, three out of eight rats presented with IMH on the T2 maps in current study. Faster T2 mapping is capable identifying the imaging characteristics as the 'striped' hypointensity in the hyperintensity infarction-core zone. This phenomenon may explain why the $\mathrm{T} 2$ value obtained by IMH was significantly lower than that in the infarction-core area and was equivalent to the $\mathrm{T} 2$ data in the sham group. Previous investigation has demonstrated that T2-mapping imaging is able to quantitatively measure the $\mathrm{T} 2$ value, which can distinguish various ischemic components (34). In the present study, T2 value in the infarction zone with IMH was significantly lower compared with that in the infarction area without IMH, which was subsequently validated by $\mathrm{H} \& \mathrm{E}$ staining outcomes. Consequently, faster T2-mapping imaging is a feasible technique to quantitatively measure IMH in a rat model with myocardial infarction. However, IMH is merely one component of varying characteristics after myocardial infarction (20), and alternative components, such as inflammation and myocardial vascular obstruction $(35,36)$, should be urgently explored by more investigations.

The present study had several limitations that should be acknowledged. First, the myocardial infarction model was assessed at only one time-point. In addition, the sample size was relatively small. Furthermore, rats with a high heart rate were examined under mechanically ventilated conditions and extrapolation of the results to human subjects should be interpreted with caution. Third, all ROIs for different segments of T2 values and myocardial infarction components were determined by two radiologists, which may have induced manual bias. It was difficult to visualize the color-coded T2 maps. Combination with other methods of CMR should therefore be recommended for detecting tissue changes after myocardial infarction.

Taken together, faster T2-mapping imaging is suitable for visualizing and quantifying myocardial AAR, IS and SMZ, and for evaluating the severity of IMH in a rat model on 7T MRI. This technique serves as a reliable non-invasive method to quantitatively assess the SMZ and IMH, contributing to monitor the viable myocardium during gene or stem cell therapy after myocardial infarction. Moreover, the mechanism of IMH will be further investigated in our subsequent research.

\section{Acknowledgements}

This study is supported by the Health and Family Planning Commission of Guizhou Province (grant no. WT-201405). The Project supported by the Postdoctoral Sustentation Fund of PLA (grant no. 20100471818) and the Ph.D. Programs Foundation of Ministry of Education of Guizhou Medical University.

\section{References}

1. Wagner A, Mahrholdt H, Thomson L, Hager S, Meinhardt G, Rehwald W, Parker M, Shah D, Sechtem U, Kim RJ and Judd RM: Effects of time, dose, and inversion time for acute myocardial infarct size measurements based on magnetic resonance imaging-delayed contrast enhancement. J Am Coll Cardiol 47: 2027-2033, 2006.

2. Ghugre NR, Ramanan V, Pop M, Yang Y, Barry J, Qiang B, Connelly KA, Dick AJ and Wright GA: Quantitative tracking of edema, hemorrhage, and microvascular obstruction in subacute myocardial infarction in a porcine model by MRI. Magn Reson Med 66: 1129-1141, 2011.

3. Zech WD, Schwendener N, Persson A, Warntjes MJ and Jackowski C: Postmortem MR quantification of the heart for characterization and differentiation of ischaemic myocardial lesions. Eur Radiol 25: 2067-2073, 2015.

4. Bönner F, Janzarik N, Jacoby C, Spieker M, Schnackenburg B, Range F, Butzbach B,Haberkorn S, Westenfeld R, Neizel-Wittke M, et al: Myocardial T2 mapping reveals age- and sex-related differences in volunteers. J Cardiovasc Magn Reson 17: 9, 2015.

5. Desch S, Engelhardt H, Meissner J, Eitel I, Sareban M, Fuernau G, de Waha S, Grothoff M, Gutberlet M, Schuler G and Thiele H: Reliability of myocardial salvage assessment by cardiac magnetic resonance imaging in acute reperfused myocardial infarction. Int J Cardiovasc Imaging 28: 263-272, 2012.

6. Kim RJ, Chen EL, Lima JA and Judd RM: Myocardial Gd-DTPA kinetics determine MRI contrast enhancement and reflect the extent and severity of myocardial injury after acute reperfused infarction. Circulation 94: 3318-3326, 1996.

7. Liu Y, Royston TJ, Klatt D and Lewandowski ED: Cardiac MR elastography of the mouse: Initial results. Magn Reson Med 76: 1879-1886, 2016.

8. Friedrich MG, Abdel-Aty H, Taylor A, Schulz-Menger J, Messroghli D and Dietz R: The salvaged area at risk in reperfused acute myocardial infarction as visualized by cardiovascular magnetic resonance. J Am Coll Cardiol 51: 1581-1587, 2008.

9. Aletras AH, Tilak GS, Natanzon A, Hsu LY, Gonzalez FM, Hoyt RF Jr and Arai AE: Retrospective determination of the area at risk for reperfused acute myocardial infarction with T2-weighted cardiac magnetic resonance imaging: Histopathological and displacement encoding with stimulated echoes, DENSE: functional validations. Circulation 113: 1865-1870, 2006.

10. Kim RJ, Wu E, Rafael A, Chen EL, Parker MA, Simonetti O, Klocke FJ, Bonow RO and Judd RM: The use of contrast-enhanced magnetic resonance imaging to identify reversible myocardial dysfunction. N Engl J Med 343: 1445-1453, 2000.

11. Higgins CB, Herfkens R, Lipton MJ, Sievers R, Sheldon P, Kaufman L and Crooks LE: Nuclear magnetic resonance imaging of acute mocardial infarction in dogs: Alterations in magnetic relaxation times. Am J Cardiol 52: 184-188, 1983.

12. Kellman $P$ and Arai AE: Imaging sequences for first pass perfusion -a review. J Cardiovas Magn Reson 9: 525-537, 2007.

13. Higgins CB and Sakuma H: Heart disease: Functional evaluation with MR imaging. Radiology 199: 307-315, 1996.

14. Verhaert D, Thavendiranathan P, Giri S, Mihai G, Rajagopalan S, Simonetti OP and Raman SV: Direct T2 quantification of myocardial edema in acute ischemic injury. JACC Cardiovasc Imaging 4: 269-278, 2011.

15. Xia R, Lu X, Zhang B, Wang Y, Liao J, Zheng J and Gao F: Assessment of myocardial edema and area at risk in a rat model of myocardial infarction with a faster T2 mapping method. Acta Radiol 56: 1085-1090, 2015.

16. Milavetz JJ, Raya TE, Johnson CS, Morkin E and Goldman S: Survival after myocardial infarction in rats: Captopril versus losartan. J Am Coll Cardiol 27: 714-719, 1996.

17. Topol EJ, Herskowitz A and Huntchins GM: Massive hemorrhage myocardial infarction after coronary thrombolysis. Am J Med 81: 339-343, 1986. 
18. Saeed M, Van T, Krug R, Hetts SW and Wilson MW: Cardiac MR imaging: Current status and future direction. Cardiovasc Diagn Ther 5: 290-310, 2015

19. Espe EK, Arosen JM, Eriksen GS, Zhang L, Smiseth OA Edvardsen T, Sjaastad I and Eriksen M: Assessment of regional myocardial work in rats. Circ Cardiovasc Imaging 8: e002695, 2015.

20. Wassmuth R, Prothemann M, Utz W, Dieringer $M$, von Knobelsdorff-Brenkenhoff F, Greiser A and Schulz-Menger J: Variability and homogeneity of cardiovascular magnetic resonance myocardial T2-mapping in volunteers compared to patients with edema. J Cardiovasc Magn Reson 15: 27, 2013.

21. Schelbert EB, Hsu LY, Anderson SA, Mohanty BD, Karim SM, Kellman P, Aletras AH and Arai AE: Late gadolinium-enhancement cardiac magnetic resonance identifies postinfarction myocardial fibrosis and the border zone at the near cellular level in ex vivo rat heart. Circ Cardiovasc Imaging 3: 743-752, 2010.

22. Feng Y, Chen F, Ma Z, Dekeyzer F, Yu J, Xie Y, Cona MM, Oyen R and Ni Y: Towards stratifying ischemic components by cardiac MRI and multifunctional stainings in a rabbit model of myocardial infarction. Theranostics 4: 24-35, 2013.

23. Langhans B, Nadjiri J, Jähnichen C, Kastrati A, Martinoff S and Hadamitzky M: Reproducibility of area at risk assessment in acute myocardial infarction by T1-and T2-mapping sequences in cardiac magnetic resonance imaging in comparison to Tc99m-sestamib SPECT. Int J Cardiovasc Imaging 30: 1357-1363, 2014.

24. Croisille P, Kim HW and Kim RJ: Controversies in cardiovascular MR imaging: T2-weighted imaging should not be used to delineate the area at risk in ischemic myocardial injury. Radiology 265: 12-22, 2012.

25. Kellman $P$ and Arai AE: Imaging sequences for first pass perfusion -a review. J cardiovasc Magn Reson 9: 525-537, 2007.

26. Giri S, Chung YC, Merchant A, Mihai G, Rajagopalan S, Raman SV and Simonetti OP: T2 quantification for improved detection of myocardial edema. J Cardiovasc Magn Reson 11: $56,2009$.

27. Verhaert D, Thavendiranathan P, Giri S, Mihai G, Rajagopalan S, Simonetti OP and Raman SV: Direct T2 quantification of myocardial edema in acute ischemic injury. JACC cardiovas Imaging 4: 269-278, 2011
28. Greenwood JP, Maredia N, Younger JF, Brown JM, Nixon J, Everett CC, Bijsterveld P, Ridgway JP, Radjenovic A, Dickinson CJ, et al: Cardiovascular magnetic resonance and single-photon emission computed tomography for diagnosis of coronary heart disease (CE-MARC): A prospective trial. Lancet 379: 453-460, 2012.

29. Mahrholdt H, Wagner A, Holly TA, Elliott MD, Bonow RO, Kim RJ and Judd RM: Reproducibility of chronic infarction size measurement by contrast-enhanced magnetic resonance imaging. Circulation 106: 2322-2327, 2002.

30. Perea Palazón RJ, Solé Arqués M, Prat González S, de Caralt Robira TM, Cibeira López MT and Ortiz Pérez JT: Parametric methods for characterizing myocardial tissue by magnetic resonance imaging (part 2): T2 mapping. Radiologia 57: 471-479, 2015 (In English, Spanish).

31. Du GQ, Du WJ, Liu JJ, Wang YS, Nie HG, Zhang MM and Yu B: Wnt1-overexpressing skeletal myoblasts as an improved cell therapy for cardiac repair following myocardial infarction. Panminerva Med 57: 153-166, 2015.

32. Yang Y, Shi C, Hou X, Zhao Y, Chen B, Tan B, Deng Z, Li Q, Liu J, Xiao Z, et al: Modified VEGF targets the ischemic myocardium and promotes functional recovery after myocardial infarction. J Control Release 213: 27-35, 2015.

33. Zhu H, Jiang X, Li X, Hu M, Wan W, Wen Y, He Y and Zheng X: Intramyocardial delivery of VEGF165 via a novel biodegradable hydrogel induces angiogenesis and improve cardiac function after rat myocardial infarction. Heart Vessels 31: 963-375, 2016.

34. Naßenstein K, Nensa F, Schlosser T, Bruder O, Umutlu L, Lauenstein T, Maderwald S and Ladd ME: Cardiac MRI: T2-mapping versus T2-weighted dark-blood TSE imaging for myocardial edema visualization in acute myocardial infarction. Rofo 186: 166-172, 2014.

35. Hausenloy DJ and Yellon DM: Time to take myocardial reperfusion injury seriously. N Engl J Med 359: 518-520, 2008.

36. Treible TA, White SK and Moon JC: Myocardial tissue characterization: Histological and pathophysiological correlation. Curr Cardiovasc Imaging Rep 7: 9254, 2014. 